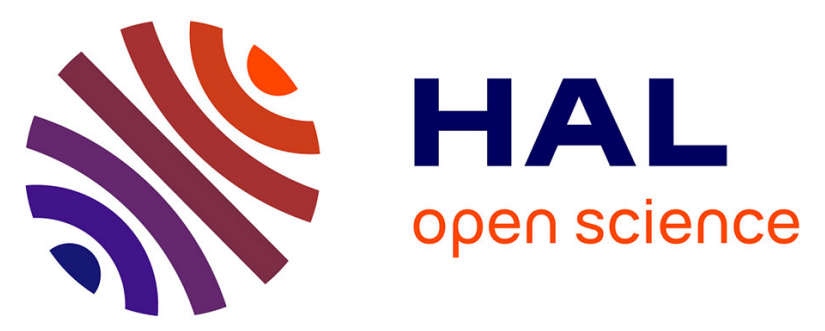

\title{
Behavior of piezoelectric layered composites with mechanical and electrical non-uniform imperfect contacts
}

\author{
J. López-Realpozo, R. Rodríguez-Ramos, A. Quintero Roba, H. \\ Brito-Santana, R. Guinovart-Díaz, Volnei Tita, Frédéric Lebon, H. \\ Camacho-Montes, Y. Espinosa-Almeyda, J. Bravo-Castillero, et al.
}

\section{To cite this version:}

J. López-Realpozo, R. Rodríguez-Ramos, A. Quintero Roba, H. Brito-Santana, R. Guinovart-Díaz, et al. Behavior of piezoelectric layered composites with mechanical and electrical non-uniform imperfect contacts. Meccanica, 2020, 55 (1), pp.125-138. 10.1007/s11012-019-01111-2 . hal-02909271

\section{HAL Id: hal-02909271 \\ https://hal.science/hal-02909271}

Submitted on 8 Sep 2020

HAL is a multi-disciplinary open access archive for the deposit and dissemination of scientific research documents, whether they are published or not. The documents may come from teaching and research institutions in France or abroad, or from public or private research centers.
L'archive ouverte pluridisciplinaire HAL, est destinée au dépôt et à la diffusion de documents scientifiques de niveau recherche, publiés ou non, émanant des établissements d'enseignement et de recherche français ou étrangers, des laboratoires publics ou privés. 


\title{
Behavior of piezoelectric layered composites with mechanical and electrical non-uniform imperfect contacts
}

\author{
J. C. López-Realpozo - R. Rodríguez-Ramos (1) A. J. Quintero Roba • \\ H. Brito-Santana - R. Guinovart-Díaz $\cdot$ Volnei Tita $\cdot$ F. Lebon • \\ H. Camacho-Montes $\cdot$ Y. Espinosa-Almeyda $\cdot$ J. Bravo-Castillero $\cdot$ \\ F. J. Sabina
}

\begin{abstract}
In this work, the two scales asymptotic homogenization method (AHM) is applied for determining the effective coefficients of laminated piezoelectric composite with periodic structure under nonuniform electrical and mechanical imperfect contact conditions. The analytical expressions of the local problems and the effective coefficients as result of the AHM are explicitly described. The constituent materials have properties belonging to $2 \mathrm{~mm}$ symmetry point group. Numerical values of the effective coefficients are reported and compared with limit cases, where perfect and uniform imperfect contact conditions are considered. Good agreements are found for
\end{abstract}

J. C. López-Realpozo · R. Rodríguez-Ramos ( $₫)$.

A. J. Quintero Roba · R. Guinovart-Díaz

Facultad de Matemática y Computación, Universidad de

La Habana, San Lázaro y L, 10400 La Habana, Cuba

e-mail: reinaldo@matcom.uh.cu

H. Brito-Santana

Department of Mathematics, Metropolitan University of Technology, Las Palmeras 3360, Nuñoa,

Santiago de Chile, Chile

\section{Tita}

Department of Aeronautical Engineering, Sao Carlos

School of Engineering, University of São Paulo, Av. João

Dagnone 1100, São Carlos, SP, Brazil

F. Lebon

Laboratoire de mécanique et d'acoustique, 4 impasse

Nikola Tesla, Cs 40006, 13453 Marseille Cedex 13,

Marseille, France these comparisons. Hence, the effect of the nonuniform imperfect contact conditions on the effective coefficients can be analyzed.

Keywords Piezoelectric laminate composite · Asymptotic homogenization method · Non-uniform imperfect contact $\cdot 2 \mathrm{~mm}$ material symmetry

H. Camacho-Montes

Instituto de Ingeniería y Tecnología, Universidad Autónoma de Ciudad Juárez, Av. del Charro 610 Norte, 32310 Ciudad Juárez, Chihuahua, México

Y. Espinosa-Almeyda · J. Bravo-Castillero · F. J. Sabina Instituto de Investigaciones en Matemáticas Aplicadas y en Sistemas, Universidad Nacional Autónoma de México, Apartado Postal 20-126, Alcaldía Álvaro Obregón, 01000 CDMX, México 


\section{Introduction}

Several piezoelectric composites can be made by combining a piezoelectric ceramic with a passive polymer phase. The properties of piezoelectric composites depend on constituent's properties, the interphase bonding conditions and the different phase arrangements of the composites. The effect of the interphase conditions on the mechanical and physical properties has attracted a great deal of researcher's attention.

The prediction of the effective coefficients taking into account different interfaces is the fundamental problems in mechanics of composites [1-5]. The adhesively bonded joints are very used in applications in the aeronautical, automotive and many other industries where prime requirements to use composite made with light weight panels and high fatigue strength are issues of interest. Different authors have investigated the adhesive joints condition [6-11]. Several works [12-16] have shown that epoxy adhesives form a so-called interphase in adhesive joints. Hence, the term interphase to refer to an interlayer is used in the present article.

As expected, the effective coefficients depend on the microstructures and properties of the layers, but also the interfacial bonding conditions need to be considered. A number of works are focused on multilayered with imperfect interfaces where the effective properties have been calculated by considering the interface effect at the micro-scale level $[1,2,5,17]$ or irregular interphases [18], i.e., the influence of the interface between both constituents on the effective properties of a composite material.

Different techniques have been used to estimate the effective properties of composites materials; the twoscale asymptotic expansion method [19, 20] was applied by Galka et al. [21] to compute macro behavior in thermo-piezoelectric composites. Further research activities have focused on studies on the micro-scale, where different approaches [22-30] have been considered for describing perfect and imperfect adhesion with a uniform interface between the constituents. A mathematical structure was developed to calculate the mechanical behavior of inhomogeneous media under the statement of an ordered microstructure with perfect contact. In Refs. [23] and [24], it was proposed a two scales asymptotic expansion for the homogenization equations considering a perfect contact conditions between the constituents. The interfaces between both materials have been described considering a uniform spring parameter, as can be seen in Refs. [25-28].

In this work, the AHM is applied for determining the effective coefficients of laminated piezoelectric composite with periodic structure and considering non-uniform electrical and mechanical imperfect contacts. The heterogeneous medium with a structure at two length scales, macroscopic and microscopic can be simulated by a homogeneous medium depending on the homogenized or effective properties. It is well known that under the assumptions of periodicity and the strict separation of scales, the behavior of composites is completely determined by the solution of the so-called local problems based on the period of the composite [23, 24]. The theoretical details of AHM have been rigorously developed in previous studies, e.g., Refs. [19, 20, 22, 23, 30]. The general method to calculate the effective properties is performed assuming the point group $2 \mathrm{~mm}$ for material symmetry. The effective properties of layered composites considering non-uniform imperfect adhesion has been investigated in [31], only considering elastic laminated composites. Thus, the present work is an extension of previous results where piezoelectric constituents are incorporated. In addition, two types of possible contact imperfections are considered: (1) mechanical imperfection simulated by spring type, i.e. the stresses are proportional to the jump of the mechanical displacements at the interface, and (2) electrical imperfection, in which the dielectric displacement is proportional to the jump of electrical potential at the interface.

\section{Formulation of the problem}

Let us consider a bounded periodically laminated piezoelectric composite $\Omega \subset \mathbb{R}^{3}$ with boundary $\partial \Omega$ in the Cartesian system of coordinates $\left\{\mathrm{x}_{1}, \mathrm{x}_{2}, \mathrm{x}_{3}\right\}$, as shown in Fig. 1a. The region $\Omega$ is defined as a parallelepiped generated by repetitions of the periodic cell Y (see, Fig. 1b), in which the layered direction is along the $\mathrm{x}_{3}$ axis. The piezoelectric constituents have properties belonging to $2 \mathrm{~mm}$ symmetry point group. The associated periodic cell $\mathrm{Y}$ is defined as $\mathrm{Y}=$ $\left\{\left(\mathrm{y}_{1}, \mathrm{y}_{2}, \mathrm{y}_{3}\right) \in \mathbb{R}^{3}: 0<\mathrm{y}_{\mathrm{i}}<\mathrm{l}_{\mathrm{i}}\right\}$ with $\mathrm{i}=1,2,3$ at the 


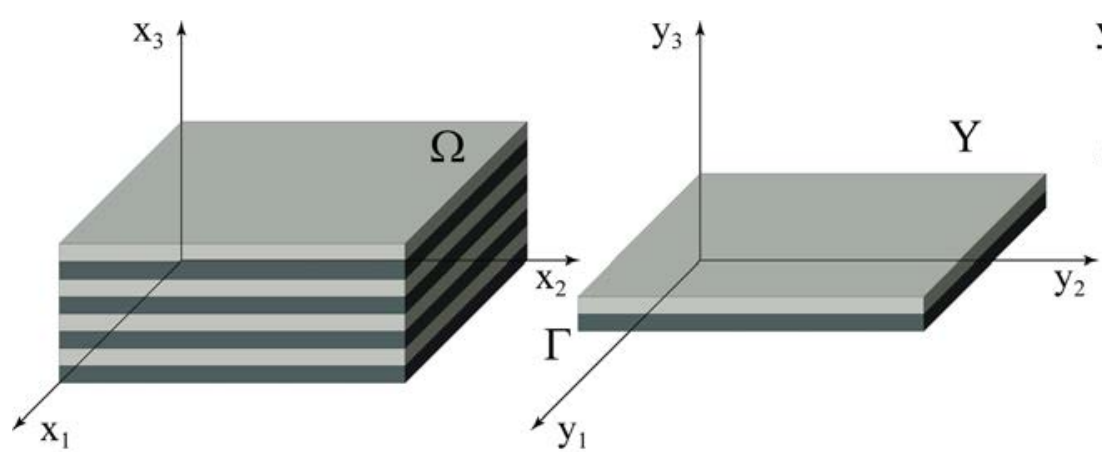

(a)

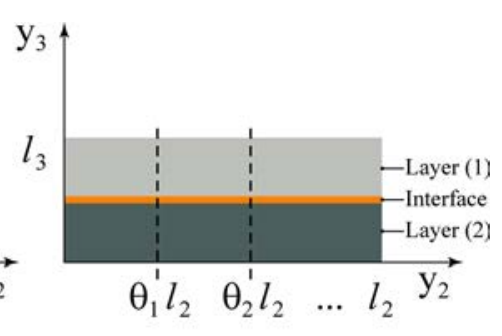

(b) (c)

Fig. 1 a Laminate composite $\Omega$, b Periodic cell Y, c Partition of the interface $\Gamma$

microscale level, in the Cartesian system of coordinates $\left\{\mathrm{y}_{1}, \mathrm{y}_{2}, \mathrm{y}_{3}\right\}$.

Herein, the periodic cell $\mathrm{Y}$ is characterized by a bilaminated composite where the non-uniform imperfect interface region between the layers is denoted by $\Gamma$ (see, Fig. 1b). Figure 1c shows the cross-section of the periodic cell $\mathrm{Y}$. The contact region $\Gamma$ is partitioned along $\mathrm{y}_{2}$ direction in subregions $\Gamma_{\mathrm{r}}$, where each $\Gamma_{\mathrm{r}}$ region has uniform imperfection parameters. In addition, $\theta_{\mathrm{r}} l_{2}$ is defined as the length of the r-partition where $\theta_{\mathrm{r}}(\mathrm{r}=1,2, \ldots, \mathrm{N} ; \mathrm{N} \in \mathbb{N})$ is the length fraction of imperfection. $\mathrm{N}$ is the number of the partitions of the interface $\Gamma$. We also have that $\sum_{\mathrm{r}=1}^{\mathrm{N}} \theta_{\mathrm{r}}=100 \%$ and $\cup \Gamma_{\mathrm{r}}=\Gamma$.

The AHM is applied to periodic layered composite (see, Fig. 1) under non-uniform imperfect contact. A general field variable $f_{i}$ now depends on both the macro-scale or fast variable " $\mathbf{x}$ " and micro-scale or slow variable " $\mathbf{y}$ ", i.e., $\mathrm{f}_{\mathrm{i}}=\mathrm{f}_{\mathrm{i}}(\mathbf{x}, \mathbf{y})$ where $\mathbf{y}=\mathbf{x} / \varepsilon$ with $\varepsilon=l / \mathrm{L}$ such that $l$ and $\mathrm{L}$ are the characteristic lengths of the periodic cell $\mathrm{Y}$ and the parallelepiped composite, respectively. Hence, the partial derivatives take the form:

$\partial \mathrm{f}_{\mathrm{i}} / \partial \mathrm{x}_{\mathrm{j}}=\partial \mathrm{f}_{\mathrm{i}} / \partial \mathrm{x}_{\mathrm{j}}+\varepsilon^{-1} \partial \mathrm{f}_{\mathrm{i}} / \partial \mathrm{y}_{\mathrm{j}}$.

Then, assuming that the body forces and free charge density are equal to zero, the piezoelectric equilibrium equations are given by

$\sigma_{\mathrm{ij}, \mathrm{j}}=0, \quad \mathrm{D}_{\mathrm{i}, \mathrm{i}}=0, \quad$ in $\mathrm{Y}$,

considering $(\cdot)_{\mathrm{j}}=\partial(\cdot) / \partial \mathrm{x}_{\mathrm{j}}$, and the constitutive equations for piezoelectric materials by components are:

$$
\sigma_{\mathrm{ij}}=\mathrm{C}_{\mathrm{ijk} \mathrm{l}} \mathrm{u}_{\mathrm{k}, \mathrm{l}}+\mathrm{e}_{\mathrm{kij}} \varphi_{, \mathrm{k}}, \quad \mathrm{D}_{\mathrm{i}}=\mathrm{e}_{\mathrm{ikl}} \mathrm{u}_{\mathrm{k}, \mathrm{l}}-\mathrm{d}_{\mathrm{ik}} \varphi_{, \mathrm{k}},
$$

where $\sigma_{\mathrm{ij}}, \mathrm{u}_{\mathrm{k}}, \mathrm{D}_{\mathrm{i}}$ and $\varphi$ are the components of the stress tensor, the mechanical and electrical displacement vectors, and the scalar electric potential, respectively. Herein, the Latin indices $\mathrm{i}, \mathrm{j}, \mathrm{k}, 1$ take values 1,2,3.

Besides, the boundary conditions can be written in the form:

$\left.\sigma_{\mathrm{ij}} \mathrm{n}_{\mathrm{j}}\right|_{\Gamma_{\mathrm{r}}}={ }^{\mathrm{r}} \mathrm{K}_{\mathrm{ij}}\left[\left[\mathrm{u}_{\mathrm{j}}\right]\right]_{\Gamma_{\mathrm{r}}}, \quad\left[\left[\sigma_{\mathrm{ij}}\right]\right]_{\Gamma_{\mathrm{r}}} \mathrm{n}_{\mathrm{j}}=0, \quad$ on $\Gamma_{\mathrm{r}}$,

$\mathrm{D}_{\mathrm{i}} \mathrm{n}_{\mathrm{i}}={ }^{\mathrm{r}} \mathrm{M}[[\varphi]]_{\Gamma_{\mathrm{r}}}, \quad\left[\left[\mathrm{D}_{\mathrm{i}}\right]\right]_{\Gamma_{\mathrm{r}}} \mathrm{n}_{\mathrm{i}}=0 \quad$ on $\Gamma_{\mathrm{r}}$,

where ${ }^{\mathrm{r}} \mathrm{K}_{\mathrm{ij}}=0$ if $\mathrm{i} \neq \mathrm{j}$, and $\Gamma$ satisfy that $\Gamma=\bigcup_{\mathrm{r}=1}^{\mathrm{N}} \Gamma_{\mathrm{r}}$.

Herein, ${ }^{\mathrm{r}} \mathrm{K}_{\mathrm{ij}}$ and ${ }^{\mathrm{r}} \mathrm{M}$ denote the mechanical and electrical imperfection parameters, respectively, for the r-interface partition $\Gamma_{\mathrm{r}}$ in $\mathrm{Y}_{\mathrm{r}}$ region partition of $\mathrm{Y}$, with $r=1,2, \ldots, N, \bigcup_{N} Y_{r}=Y$. Herein, the symbol $Y_{r}$ describes the r-partition of the periodic cell $Y$, the notations $[[\cdot]]=(\cdot)^{(1)}-(\cdot)^{(2)}$ represents the jump across the interface $\Gamma$.

The imperfect contacts are modeled considering a layer of zero thickness where a spring describes the mechanical imperfection, and a capacitor can be related to the electrical imperfection. The tangential and normal components of the mechanical imperfection parameters are defined as $\mathrm{K}_{\mathrm{t}}=\mathrm{K}_{11}, \mathrm{~K}_{\mathrm{s}}=\mathrm{K}_{22}$ and $\mathrm{K}_{\mathrm{n}}=\mathrm{K}_{33}$, whereas $\mathrm{M}$ is the electrical imperfection parameter. The infinite value for the imperfection parameters implies vanishing of the imperfection (the so called perfect interface conditions) and the zero values for the imperfection parameters imply debonding. Any finite positive values for the interface parameters define an imperfect interface, see Ref. [24]. 
Using the asymptotic expansion, the mechanical displacements and the electric potential are written as:

$\mathrm{u}_{\mathrm{i}}(\mathbf{x})=\mathrm{u}_{\mathrm{i}}^{(0)}(\mathbf{x})+\sum_{\mathrm{k}=1}^{\infty} \varepsilon^{\mathrm{k}} \mathrm{u}_{\mathrm{i}}^{(\mathrm{k})}(\mathbf{x}, \mathbf{y})$,

$\varphi(\mathbf{x})=\varphi^{(0)}(\mathbf{x})+\sum_{\mathrm{k}=1}^{\infty} \varepsilon^{\mathrm{k}} \varphi^{(\mathrm{k})}(\mathbf{x}, \mathbf{y})$.

Then, if Eq. (6) is substituted into Eqs. (2)-(5), and further, Eq. (1) and the expressions

$$
\begin{aligned}
& u_{\mathrm{i}}^{(\mathrm{k})}(\mathbf{x}, \mathbf{y}) \equiv \Phi_{\mathrm{imn}}^{(\mathrm{k})} \frac{\partial^{\mathrm{k}} \mathrm{u}_{\mathrm{m}}^{(0)}}{\partial \mathrm{x}_{\mathrm{n}}^{\mathrm{k}}}+\Pi_{\mathrm{im}}^{(\mathrm{k})} \frac{\partial^{\mathrm{k}} \varphi^{(0)}}{\partial \mathrm{x}_{\mathrm{m}}^{\mathrm{k}}}, \\
& \varphi^{(\mathrm{k})}(\mathbf{x}, \mathbf{y}) \equiv \Psi_{\mathrm{mn}}^{(\mathrm{k})} \frac{\partial^{\mathrm{k}} \mathrm{u}_{\mathrm{m}}^{(0)}}{\partial \mathrm{x}_{\mathrm{n}}^{\mathrm{k}}}+\Theta_{\mathrm{m}}^{(\mathrm{k})} \frac{\partial^{\mathrm{k}} \varphi^{(0)}}{\partial \mathrm{x}_{\mathrm{m}}^{\mathrm{k}}},
\end{aligned}
$$

where $\Phi_{\mathrm{imn}}^{(\mathrm{k})}(\mathbf{y}), \Pi_{\mathrm{im}}^{(\mathrm{k})}(\mathbf{y}), \quad \Psi_{\mathrm{mn}}^{(\mathrm{k})}(\mathbf{y})$, and $\Theta_{\mathrm{m}}^{(\mathrm{k})}(\mathbf{y})$ are periodic functions with a periodic length equal to $l$. It is possible to determine equivalent expressions to those reported in Ref. [31] for the elastic case. Therefore, different equations are obtained which depends on the $\varepsilon$ parameter. Subsequently, if the terms are grouped according to the order of $\varepsilon$ $\left(\varepsilon^{-2}, \varepsilon^{-1}, \varepsilon^{0}, \ldots\right)$, and appropriate conditions are taking into account in order to guarantee the existence of the $l$-periodic solutions. Then, a recurrent family of partial differential equations is obtained. This way, the expressions of the local problems on $\mathrm{Y}$, the equivalent homogenized problems and the corresponding effective coefficients can be stated. Theoretical details of the rigorous mathematical foundation of the AHM can be found in Refs. [19, 20, 22, 23], and here are omitted.

Hence, from the terms of the $\varepsilon^{-1}$ order, the partial derivate equations are obtained:

$$
\left\{\begin{array}{l}
\frac{\partial}{\partial \mathrm{y}_{\mathrm{j}}}\left[\mathrm{C}_{\mathrm{ijpq}}(\mathbf{y})+\mathrm{C}_{\mathrm{ijk} l}(\mathrm{y}) \frac{\partial^{\mathrm{r}} \Phi_{\mathrm{kpq}}(\mathbf{y})}{\partial \mathrm{y}_{l}}+\mathrm{e}_{l \mathrm{ij}}(\mathrm{y}) \frac{\partial^{\mathrm{r}} \Psi_{\mathrm{pq}}(\mathbf{y})}{\partial \mathrm{y}_{l}}\right]=0, \\
\frac{\partial}{\partial \mathrm{y}_{\mathrm{j}}}\left[\mathrm{e}_{\mathrm{pij}}(\mathbf{y})+\mathrm{C}_{\mathrm{ijk} l}(\mathrm{y}) \frac{\partial^{\mathrm{r}} \Pi_{\mathrm{kp}}(\mathbf{y})}{\partial \mathrm{y}_{l}}+\mathrm{e}_{l \mathrm{ij}}(\mathrm{y}) \frac{\partial^{\mathrm{r}} \Theta_{\mathrm{p}}(\mathbf{y})}{\partial \mathrm{y}_{l}}\right]=0, \\
\frac{\partial}{\partial \mathrm{y}_{\mathrm{j}}}\left[\mathrm{e}_{\mathrm{jpq}}(\mathbf{y})+\mathrm{e}_{\mathrm{jk} l}(\mathrm{y}) \frac{\partial^{\mathrm{r}} \Phi_{\mathrm{kpq}}(\mathbf{y})}{\partial \mathrm{y}_{l}}-\mathrm{d}_{\mathrm{j} l}(\mathrm{y}) \frac{\partial^{\mathrm{r}} \Psi_{\mathrm{pq}}(\mathbf{y})}{\partial \mathrm{y}_{l}}\right]=0, \\
\frac{\partial}{\partial \mathrm{y}_{\mathrm{j}}}\left[\mathrm{d}_{\mathrm{jp}}(\mathbf{y})-\mathrm{e}_{\mathrm{jk} l}(\mathrm{y}) \frac{\partial^{\mathrm{r}} \Pi_{\mathrm{kp}}(\mathbf{y})}{\partial \mathrm{y}_{l}}+\mathrm{d}_{\mathrm{j} l}(\mathrm{y}) \frac{\partial^{\mathrm{r}} \Theta_{\mathrm{p}}(\mathbf{y})}{\partial \mathrm{y}_{l}}\right]=0 .
\end{array}\right.
$$

Consequently, from the terms of the $\varepsilon^{0}$ order, the equivalent homogenized equations of the problem Eq. (2) are determined

$$
\left\{\begin{array}{l}
C_{i j p q}^{*} \frac{\partial^{2} u_{p}^{(0)}}{\partial y_{j} \partial y_{q}}+e_{p i j}^{*} \frac{\partial^{2} \varphi^{(0)}}{\partial y_{p} \partial y_{j}}=0 \\
e_{i j p}^{*} \frac{\partial^{2} u_{p}^{(0)}}{\partial y_{i} \partial y_{j}}-d_{i p}^{*} \frac{\partial^{2} \varphi^{(0)}}{\partial y_{i} \partial y_{p}}=0
\end{array},\right.
$$

with effective coefficients in the form

$$
\left\{\begin{array}{l}
\mathrm{C}_{\mathrm{ijpq}}^{*}=\sum_{\mathrm{r}=1}^{\mathrm{N}}\left\langle\mathrm{C}_{\mathrm{ijpq}}+\mathrm{C}_{\mathrm{ijk} l} \frac{\partial^{\mathrm{r}} \Phi_{\mathrm{kpq}}}{\partial y_{l}}+\mathrm{e}_{l \mathrm{ij}} \frac{\partial^{\mathrm{r}} \Psi_{\mathrm{pq}}}{\partial y_{l}}\right\rangle, \\
\mathrm{e}_{\mathrm{ipq}}^{*}=\sum_{\mathrm{r}=1}^{\mathrm{N}}\left\langle\mathrm{e}_{\mathrm{ipq}}+\mathrm{e}_{\mathrm{ik} l} \frac{\partial^{\mathrm{r}} \Phi_{\mathrm{kpq}}}{\partial y_{l}}-\mathrm{d}_{\mathrm{i} l} \frac{\partial^{\mathrm{r}} \Psi_{\mathrm{pq}}}{\partial y_{l}}\right\rangle, \\
\mathrm{e}_{\mathrm{pij}}^{*}=\sum_{\mathrm{r}=1}^{\mathrm{N}}\left\langle\mathrm{e}_{\mathrm{pij}}+\mathrm{C}_{\mathrm{ijk} l} \frac{\partial^{\mathrm{r}} \Pi_{\mathrm{kp}}}{\partial y_{l}}+\mathrm{e}_{l i j} \frac{\partial^{\mathrm{r}} \Theta_{\mathrm{p}}}{\partial y_{l}}\right\rangle, \\
\mathrm{d}_{\mathrm{ip}}^{*}=\sum_{\mathrm{r}=1}^{\mathrm{N}}\left\langle\mathrm{d}_{\mathrm{ip}}-\mathrm{e}_{\mathrm{ik} l} \frac{\partial^{\mathrm{r}} \Pi_{\mathrm{kp}}}{\partial y_{l}}+\mathrm{d}_{\mathrm{i} l} \frac{\partial^{\mathrm{r}} \Theta_{\mathrm{p}}}{\partial y_{l}}\right\rangle .
\end{array}\right.
$$

As mentioned above, constituent's distribution periodicity is along the $y_{3}$ direction. However, the distribution of the imperfection parameters at the interface $\Gamma$ is directed in the $y_{2}$ axes. From the mathematical point of view, this situation must be treated as a $2 \mathrm{D}$ problem written with partial derivates, see Ref. [31]. Imperfection region $\Gamma$ is divided in $\mathrm{N}$ partitions $\Gamma_{\mathrm{r}}$ and each one has a uniform imperfection parameters, then, the functions $\Phi_{\mathrm{imn}}^{(\mathrm{k})}(\mathbf{y}), \Pi_{\mathrm{im}}^{(\mathrm{k})}(\mathbf{y})$, $\Psi_{\mathrm{mn}}^{(\mathrm{k})}(\mathbf{y})$, and $\Theta_{\mathrm{m}}^{(\mathrm{k})}(\mathbf{y})$ can be proposed as a piecewise linear function in $\mathrm{Y}$ defined as:

$$
\begin{aligned}
& \Phi_{\mathrm{imn}}^{(\mathrm{k})}(\mathbf{y})=\left\{\begin{array}{rc}
{ }^{1} \Phi_{\mathrm{imn}}^{(\mathrm{k})}(\mathbf{y}) & \text { in } Y_{1} \\
\vdots & \\
{ }^{\mathrm{N}} \Phi_{\mathrm{imn}}^{(\mathrm{k})}(\mathbf{y}) & \text { in } Y_{\mathrm{N}}
\end{array}\right. \\
& \Pi_{\mathrm{im}}^{(\mathrm{k})}(\mathbf{y})=\left\{\begin{array}{rc}
{ }^{1} \Pi_{\mathrm{im}}^{(\mathrm{k})}(\mathbf{y}) & \text { in } Y_{1} \\
\vdots & \\
{ }^{\mathrm{N}} \Pi_{\mathrm{im}}^{(\mathrm{k})}(\mathbf{y}) & \text { in } Y_{\mathrm{N}}
\end{array}\right. \\
& \Psi_{\mathrm{mn}}^{(\mathrm{k})}(\mathbf{y})=\left\{\begin{array}{rc}
{ }^{1} \Psi_{\mathrm{mn}}^{(\mathrm{k})}(\mathbf{y}) & \text { in } Y_{1} \\
\vdots & \\
{ }^{\mathrm{N}} \Psi_{\mathrm{mn}}^{(\mathrm{k})}(\mathbf{y}) & \text { in } Y_{\mathrm{N}}
\end{array}\right.
\end{aligned}
$$

and $\Theta_{\mathrm{m}}^{(\mathrm{k})}(\mathbf{y})=\left\{\begin{aligned}{ }^{1} \Theta_{\mathrm{m}}^{(\mathrm{k})}(\mathbf{y}) & \text { in } Y_{1} \\ \vdots & \\ { }^{\mathrm{N}} \Theta_{\mathrm{m}}^{(\mathrm{k})}(\mathbf{y}) & \text { in } Y_{\mathrm{N}}\end{aligned}\right.$. 
This way, local problems can be solved for each rpartition. As the imperfection parameter is constant for each partition, no dependence on $\mathrm{y}_{2}$ needs to be considered and the local problems equations only depends on $y_{3}$.

Therefore, the local problems ${ }_{p q} \mathrm{~L}$ and ${ }_{\mathrm{p}} \mathrm{I}$ for each $\mathrm{r}$ interphase partition are written as:

${ }_{\mathrm{pq}} \mathrm{L}$ problem

$\left\{\begin{array}{l}\frac{\mathrm{d}}{\mathrm{dy}_{3}}\left[\mathrm{C}_{\mathrm{i} 3 \mathrm{pq}}(\mathbf{y})+C_{\mathrm{i} 3 \mathrm{k} 3}(\mathrm{y}) \frac{\mathrm{d}^{\mathrm{r}} \Phi_{\mathrm{kpq}}(\mathbf{y})}{\mathrm{dy}_{3}}+\mathrm{e}_{3 \mathrm{ij}}(\mathrm{y}) \frac{\mathrm{d}^{\mathrm{r}} \Psi_{\mathrm{pq}}(\mathbf{y})}{\mathrm{dy}_{3}}\right]=0, \\ \frac{\mathrm{d}}{\mathrm{dy}_{3}}\left[e_{3 \mathrm{pq}}(\mathbf{y})+e_{3 \mathrm{k} 3}(\mathrm{y}) \frac{\mathrm{d}^{\mathrm{r}} \Phi_{\mathrm{kpq}}(\mathbf{y})}{\mathrm{dy}_{3}}-\mathrm{d}_{33}(\mathrm{y}) \frac{\mathrm{d}^{\mathrm{T}} \Psi_{\mathrm{pq}}(\mathbf{y})}{\mathrm{dy}_{3}}\right]=0,\end{array}\right.$ in $\mathrm{Y}_{\mathrm{r}}$

with interface conditions

$$
\begin{aligned}
& \left\{\left[\left[\mathrm{C}_{\mathrm{i} 3 \mathrm{pq}}+\mathrm{C}_{\mathrm{i} 3 \mathrm{k} 3} \frac{\mathrm{d}^{\mathrm{r}} \Phi_{\mathrm{kpq}}}{\mathrm{dy}_{3}}+\mathrm{e}_{3 \mathrm{i} 3} \frac{\mathrm{d}^{\mathrm{r}} \Psi_{\mathrm{pq}}}{\mathrm{dy}_{3}}\right]\right] \mathrm{n}_{3}=0\right. \\
& \left\{\left[\left[e_{3 p q}+e_{3 k 3} \frac{d^{r} \Phi_{k p q}}{d y_{3}}-d_{33} \frac{d^{r} \Psi_{p q}}{d y_{3}}\right]\right] n_{3}=0,\right. \\
& \left\langle{ }^{\mathrm{r}} \Phi_{\mathrm{kpq}}\right\rangle=0 \text { and }\left\langle{ }^{\mathrm{r}} \Psi_{\mathrm{pq}}\right\rangle=0 . \\
& \left\{\begin{array}{l}
\frac{d}{d y_{3}}\left[e_{\mathrm{pi} 3}(\mathbf{y})+C_{\mathrm{izk}}(\mathrm{y}) \frac{\mathrm{d}^{\mathrm{r}} \Pi_{\mathrm{kp}}(\mathbf{y})}{\mathrm{dy}_{3}}+\mathrm{e}_{3 \mathrm{iz}}(\mathrm{y}) \frac{\mathrm{d}^{\mathrm{r}} \Theta_{\mathrm{p}}(\mathbf{y})}{\mathrm{dy}_{3}}\right]=0, \\
\frac{\mathrm{d}}{\mathrm{dy}_{3}}\left[\mathrm{~d}_{3 \mathrm{p}}(\mathbf{y})-\mathrm{e}_{3 \mathrm{k} 3}(\mathrm{y}) \frac{\mathrm{d}^{\mathrm{r}} \Pi_{\mathrm{kp}}(\mathbf{y})}{\mathrm{dy}_{3}}+\mathrm{d}_{33}(\mathrm{y}) \frac{\mathrm{d}^{\mathrm{r}} \Theta_{\mathrm{p}}(\mathbf{y})}{\mathrm{dy}_{3}}\right]=0 .
\end{array}\right.
\end{aligned}
$$

with interface conditions

$$
\left\{\begin{array}{l}
{\left[\left[e_{\mathrm{pi} 3}+\mathrm{C}_{\mathrm{i} 3 \mathrm{k} 3} \frac{\mathrm{d}^{\mathrm{r}} \Pi_{\mathrm{kp}}}{\mathrm{dy}_{3}}+\mathrm{e}_{3 \mathrm{i} 3} \frac{\mathrm{d}^{\mathrm{r}} \Theta_{\mathrm{p}}}{\mathrm{dy}_{3}}\right]\right] \mathrm{n}_{3}=0,} \\
{\left[\left[-\mathrm{d}_{3 \mathrm{p}}+\mathrm{e}_{3 \mathrm{k} 3} \frac{\mathrm{d}^{\mathrm{r}} \Pi_{\mathrm{kp}}}{\mathrm{dy}_{3}}-\mathrm{d}_{33} \frac{\mathrm{d}^{\mathrm{r}} \Theta_{\mathrm{p}}}{\mathrm{dy}_{3}}\right]\right] \mathrm{n}_{3}=0,}
\end{array}\right.
$$

$$
\begin{cases}\left.\left(\mathrm{e}_{\mathrm{p} i 3}+\mathrm{C}_{\mathrm{i} 3 \mathrm{k} 3} \frac{\mathrm{d}^{\mathrm{r}} \Pi_{\mathrm{kp}}}{\mathrm{dy}_{3}}+\mathrm{e}_{3 \mathrm{ij}} \frac{\mathrm{d}^{\mathrm{r}} \Theta_{\mathrm{p}}}{\mathrm{dy}_{3}}\right) \mathrm{n}_{3}={ }^{\mathrm{r}} \mathrm{K}_{\mathrm{ik}}\left[{ }^{\mathrm{r}} \Pi_{\mathrm{kp}}\right]\right], & \\ \left(-\mathrm{d}_{3 \mathrm{p}}+\mathrm{e}_{3 \mathrm{k} 3} \frac{\mathrm{d}^{\mathrm{r}} \Pi_{\mathrm{kp}}}{\mathrm{dy}_{3}}-\mathrm{d}_{33} \frac{\mathrm{d}^{\mathrm{r}} \Theta_{\mathrm{p}}}{\mathrm{dy}_{3}}\right) \mathrm{n}_{3}={ }^{\mathrm{r}} \mathrm{M}\left[\left[{ }^{\mathrm{r}} \Theta_{\mathrm{p}}\right]\right] . & \text { on } \Gamma_{\mathrm{r}}\end{cases}
$$

$\left\langle{ }^{\mathrm{r}} \Pi_{\mathrm{kp}}\right\rangle=0$ and $\left\langle{ }^{\mathrm{r}} \Theta_{\mathrm{p}}\right\rangle=0$.

As can be seem, the local problems ${ }_{\mathrm{pq}} \mathrm{L}$ [Eqs. (12)(15)] and ${ }_{p} \mathrm{I}$, [Eqs. (16)-(19)] result from the fact that unknown functions group in two separate set $\left(\mathrm{d}^{\mathrm{r}} \Phi_{\mathrm{kpq}}^{(1)}(\mathbf{y}) / \mathrm{dy}_{3}, \mathrm{~d}^{\mathrm{r}} \Psi_{\mathrm{pq}}^{(1)}(\mathbf{y}) / \mathrm{dy}_{3}\right) \quad$ and $\left(\mathrm{d}^{\mathrm{r}} \Pi_{\mathrm{kp}}^{(1)}(\mathbf{y}) / \mathrm{dy}_{3}, \mathrm{~d}^{\mathrm{r}} \Theta_{\mathrm{p}}^{(1)}(\mathbf{y}) / \mathrm{dy}_{3}\right)$. Also, the average operator $\langle\cdot\rangle=\frac{1}{|\mathrm{Y}|} \int_{\mathrm{Y}}(\cdot) \mathrm{dY}$ represents the volume average per unit length over Y.

Consequently, the equivalent homogenized equations of the problem Eq. (2) are determined:

$$
\left\{\begin{array}{c}
C_{\mathrm{i} 3 \mathrm{p} 3}^{*} \frac{\mathrm{d}^{2} u_{\mathrm{p}}^{(0)}}{d y_{3}^{2}}+\mathrm{e}_{3 \mathrm{i} 3}^{*} \frac{\mathrm{d}^{2} \varphi^{(0)}}{d y_{3}^{2}}=0 \\
\mathrm{e}_{33 \mathrm{p}}^{*} \frac{\mathrm{d}^{2} u_{\mathrm{p}}^{(0)}}{d y_{3}^{2}}-\mathrm{d}_{33}^{*} \frac{\mathrm{d}^{2} \varphi^{(0)}}{d y_{3}^{2}}=0
\end{array},\right.
$$

with effective coefficients in the form:

${ }_{\mathrm{pq}} \mathrm{L}$ problem

$$
\left\{\begin{array}{l}
\mathrm{C}_{\mathrm{ijpq}}^{*}=\sum_{\mathrm{r}=1}^{\mathrm{N}}\left\langle C_{\mathrm{ijpq}}+\mathrm{C}_{\mathrm{ijk} 3} \frac{\mathrm{d}^{\mathrm{r}} \Phi_{\mathrm{kpq}}}{\mathrm{dy}_{3}}+\mathrm{e}_{3 \mathrm{ij}} \frac{\mathrm{d}^{\mathrm{r}} \Psi_{\mathrm{pq}}}{\mathrm{dy}_{3}}\right\rangle, \\
\mathrm{e}_{\mathrm{ipq}}^{*}=\sum_{\mathrm{r}=1}^{\mathrm{N}}\left\langle\mathrm{e}_{\mathrm{ipq}}+\mathrm{e}_{\mathrm{ik} 3} \frac{\mathrm{d}^{\mathrm{r}} \Phi_{\mathrm{kpq}}}{\mathrm{dy}_{3}}-\mathrm{d}_{\mathrm{i} 3} \frac{\mathrm{d}^{\mathrm{r}} \Psi_{\mathrm{pq}}}{\mathrm{dy}_{3}}\right\rangle
\end{array}\right.
$$

${ }_{\mathrm{p}} \mathrm{I}$ problem

$$
\left\{\begin{array}{l}
e_{\mathrm{pij}}^{*}=\sum_{\mathrm{r}=1}^{\mathrm{N}}\left\langle\mathrm{e}_{\mathrm{pij}}+\mathrm{C}_{\mathrm{ijk} 3} \frac{\mathrm{d}^{\mathrm{r}} \Pi_{\mathrm{kp}}}{\mathrm{dy}_{3}}+\mathrm{e}_{3 \mathrm{ij}} \frac{\mathrm{d}^{\mathrm{r}} \Theta_{\mathrm{p}}}{\mathrm{dy}_{3}}\right\rangle, \\
\mathrm{d}_{\mathrm{ip}}^{*}=\sum_{\mathrm{r}=1}^{\mathrm{N}}\left\langle\mathrm{d}_{\mathrm{ip}}-\mathrm{e}_{\mathrm{ik} 3} \frac{\mathrm{d}^{\mathrm{r}} \Pi_{\mathrm{kp}}}{\mathrm{dy}_{3}}+\mathrm{d}_{\mathrm{i} 3} \frac{\mathrm{d}^{\mathrm{r}} \Theta_{\mathrm{p}}}{\mathrm{dy}_{3}}\right\rangle .
\end{array}\right.
$$


Then, the functions $\mathrm{d}^{\mathrm{r}} \Phi_{\mathrm{kpq}}^{(1)}(\mathbf{y}) / \mathrm{dy}_{3}, \mathrm{~d}^{\mathrm{r}} \Psi_{\mathrm{pq}}^{(1)}(\mathbf{y}) / \mathrm{dy}_{3}$, $\mathrm{d}^{\mathrm{r}} \Pi_{\mathrm{kp}}^{(1)}(\mathbf{y}) / \mathrm{dy}_{3}$ and $\mathrm{d}^{\mathrm{r}} \Theta_{\mathrm{p}}^{(1)}(\mathbf{y}) / \mathrm{dy}_{3}$ need to be determine for each local problems.

Integrating Eqs. (12) and (16) respect to $y_{3}$, it turns out that,

$$
\begin{aligned}
& \left\{\begin{array}{l}
\mathrm{C}_{\mathrm{i} 3 \mathrm{k} 3} \frac{\mathrm{d}^{\mathrm{r}} \Phi_{\mathrm{kpq}}^{(1)}}{\mathrm{dy}_{3}}+\mathrm{e}_{3 \mathrm{i} 3} \frac{\mathrm{d}^{\mathrm{r}} \Psi_{\mathrm{pq}}^{(1)}}{\mathrm{dy}_{3}}=\mathrm{A}_{\mathrm{i} 3 \mathrm{pq}}^{\mathrm{r}}-\mathrm{C}_{\mathrm{i} 3 \mathrm{pq}}, \\
\mathrm{e}_{3 \mathrm{k} 3} \frac{\mathrm{d}^{\mathrm{r}} \Phi_{\mathrm{kpq}}^{(1)}}{\mathrm{dy}_{3}}-\mathrm{d}_{33} \frac{\mathrm{d}^{\mathrm{r}} \Psi_{\mathrm{pq}}^{(1)}}{\mathrm{dy}_{3}}=\mathrm{B}_{3 \mathrm{pq}}^{\mathrm{r}}-\mathrm{e}_{3 \mathrm{pq}},
\end{array}\right. \\
& \left\{\begin{array}{l}
\mathrm{C}_{\mathrm{i} 3 \mathrm{k} 3} \frac{\mathrm{d}^{\mathrm{r}} \Pi_{\mathrm{kp}}^{(1)}}{\mathrm{dy}_{3}}+\mathrm{e}_{3 \mathrm{i} 3} \frac{\mathrm{d}^{\mathrm{r}} \Theta_{\mathrm{p}}^{(1)}}{\mathrm{dy}_{3}}=\mathrm{J}_{\mathrm{pi} 3}^{\mathrm{r}}-\mathrm{e}_{\mathrm{pi} 3}, \\
\mathrm{e}_{3 \mathrm{k} 3} \frac{\mathrm{d}^{\mathrm{r}} \Pi_{\mathrm{kp}}^{(1)}}{\mathrm{dy}_{3}}-\mathrm{d}_{33} \frac{\mathrm{d}^{\mathrm{r}} \Theta_{\mathrm{p}}^{(1)}}{\mathrm{dy}_{3}}=\mathrm{U}_{3 \mathrm{p}}^{\mathrm{r}}+\mathrm{d}_{3 \mathrm{p}},
\end{array}\right.
\end{aligned}
$$

where $A_{i 3 p q}^{r}, B_{3 p q}^{r}, J_{3 i p}^{r}$, and $U_{3 p}^{r}$ are the integration constants that need to be found. Then, taking into account Eqs. (13), (14), (17), (18), (23), and (24), the following systems of equations are obtained:

$\left\{\begin{array}{c}{ }^{\mathrm{r}} \mathrm{Q}_{\mathrm{ji}} \mathrm{A}_{\mathrm{i} 3 \mathrm{pq}}^{\mathrm{r}}+\left\langle\mathrm{P}_{\mathrm{ji}}^{-1} \mathrm{e}_{3 \mathrm{i} 3}\right\rangle \mathrm{B}_{3 \mathrm{pq}}^{\mathrm{r}}=\left\langle\mathrm{P}_{\mathrm{ji}}^{-1}\left(\mathrm{~d}_{33} \mathrm{C}_{\mathrm{i} 3 \mathrm{pq}}+\mathrm{e}_{3 \mathrm{i} 3} \mathrm{e}_{3 \mathrm{pq}}\right)\right\rangle, \\ \left\langle\mathrm{Re}_{3 \mathrm{k} 3} \mathrm{C}_{\mathrm{k} 3 \mathrm{i} 3}^{-1}\right\rangle \mathrm{A}_{\mathrm{i} 3 \mathrm{pq}}^{\mathrm{r}}+{ }^{\mathrm{r}} \mathrm{SB}_{3 \mathrm{pq}}^{\mathrm{r}}=\left\langle\mathrm{R}\left(\mathrm{e}_{3 \mathrm{k} 3} \mathrm{C}_{\mathrm{k} 3 \mathrm{i} 3}^{-1} \mathrm{C}_{\mathrm{i} 3 \mathrm{pq}}-e_{3 \mathrm{pq}}\right)\right\rangle,\end{array}\right.$

$\left\{\begin{array}{c}{ }^{\mathrm{r}} \mathrm{Q}_{\mathrm{ki}} \mathrm{J}_{3 \mathrm{ip}}^{\mathrm{r}}+\left\langle\mathrm{P}_{\mathrm{ki}}^{-1} \mathrm{e}_{3 \mathrm{i} 3}\right\rangle \mathrm{U}_{3 \mathrm{p}}^{\mathrm{r}}=\left\langle\mathrm{P}_{\mathrm{ki}}^{-1}\left(\mathrm{~d}_{33} \mathrm{e}_{\mathrm{pi} 3}-\mathrm{e}_{3 \mathrm{i} 3} \mathrm{~d}_{3 \mathrm{p}}\right)\right\rangle, \\ \left\langle\mathrm{Re}_{3 \mathrm{k} 3} \mathrm{C}_{\mathrm{k} 3 \mathrm{i} 3}^{-1}\right\rangle \mathrm{J}_{3 \mathrm{ip}}^{\mathrm{r}}+{ }^{\mathrm{r}} \mathrm{SU}_{3 \mathrm{p}}^{\mathrm{r}}=\left\langle\mathrm{R}\left(\mathrm{e}_{3 \mathrm{k} 3} \mathrm{C}_{\mathrm{k} 3 \mathrm{i} 3}^{-1} \mathrm{e}_{\mathrm{pi} 3}+\mathrm{d}_{3 \mathrm{p}}\right)\right\rangle,\end{array}\right.$

where ${ }^{\mathrm{r}} \mathrm{Q}_{\mathrm{ji}}=\left\langle\overline{\mathrm{C}}_{\mathrm{i} 3 \mathrm{j} 3}^{-1}\right\rangle+\frac{{ }^{\mathrm{r}} \mathrm{K}_{\mathrm{ij}}^{-1}}{l_{3}}, \mathrm{P}_{\mathrm{ji}}^{-1}=\overline{\mathrm{C}}_{\mathrm{i} 3 \mathrm{j} 3}^{-1} \mathrm{~d}_{33}^{-1}, \mathrm{R}=\overline{\mathrm{d}}_{33}^{-1}$ and ${ }^{\mathrm{r}} \mathrm{S}=\frac{{ }^{\mathrm{r}} \mathrm{M}^{-1}}{l_{3}}-\left\langle\overline{\mathrm{d}}_{33}^{-1}\right\rangle$. Herein, $\langle\cdot\rangle={ }^{(1)} \mathrm{V}_{1}+{ }^{(2)} \mathrm{V}_{2}$ where $V_{1}$ and $V_{2}$ are the volume fraction of each constituent for a two-layer composite. Thus, the derived expressions $\mathrm{C}_{\mathrm{ijkl}}^{(\alpha)}, \mathrm{e}_{\mathrm{ijl}}^{(\alpha)}$ and $\mathrm{d}_{\mathrm{ij}}^{(\alpha)}$ denote the elastic, piezoelectric and dielectric material properties for each constituents, denoted as $\alpha$. The constituent $\alpha=\left\{\begin{array}{ll}1 & \text { if } 0<\mathrm{y}_{1}<\mathrm{V}_{1} l_{3}, \\ 2 & \text { if } \mathrm{V}_{1} l_{3}<\mathrm{y}_{1}<l_{3},\end{array}\right.$ where $\mathrm{V}_{1}$ is the volume fraction of layered one and $l_{3}$ is the length of the periodic cell in the $x_{3}$ direction. The magnitude $n_{j}$ is the unit vector in the outward normal direction.

Solving the systems [Eqs. (25) and (26)] and considering $2 \mathrm{~mm}$ symmetry for the composite constituents (this algorithm also works for $4 \mathrm{~mm}$ and $6 \mathrm{~mm}$ symmetry point groups), we have:

- If $\beta \neq 3$ :

${ }^{\mathrm{r}} \mathrm{A}_{\beta 3 \mathrm{pq}}=\left\langle\mathrm{C}_{\beta 3 \beta 3}^{-1} \mathrm{C}_{\beta 3 \mathrm{pq}}\right\rangle\left[\left(l_{3}{ }^{\mathrm{r}} \mathrm{K}_{\beta \beta}\right)^{-1}+\left\langle\mathrm{C}_{\beta 3 \beta 3}^{-1}\right\rangle\right]^{-1}$,

$$
{ }^{\mathrm{r}} \mathrm{J}_{\mathrm{p} \beta 3}=\left\langle\mathrm{C}_{\beta 3 \beta 3}^{-1} \mathrm{e}_{\mathrm{p} \beta 3}\right\rangle\left[\left(l_{3}{ }^{\mathrm{r}} K_{\beta \beta}\right)^{-1}+\left\langle\mathrm{C}_{\beta 3 \beta 3}^{-1}\right\rangle\right]^{-1},
$$

- If $\beta=3$ :

$$
\begin{aligned}
{ }^{\mathrm{r}} \mathrm{A}_{33 \mathrm{pq}} & ={ }^{\mathrm{r}} \mathrm{Q}^{-1}\left[\mathrm{H}_{\mathrm{pq}}-\left\langle\mathrm{P}^{-1} \mathrm{e}_{333}\right\rangle \mathrm{B}_{3 \mathrm{pq}}\right], \\
{ }^{\mathrm{r}} \mathrm{J}_{\mathrm{p} 33} & =\frac{\left\langle\mathrm{P}^{-1} \mathrm{e}_{333}\right\rangle \mathrm{N}_{\mathrm{p}}+{ }^{\mathrm{r}} \mathrm{FZ}_{\mathrm{p}}}{\left\langle\mathrm{P}^{-1} \mathrm{e}_{333}\right\rangle^{2}+{ }^{\mathrm{r}} \mathrm{F}^{\mathrm{r}} \mathrm{Q}}
\end{aligned}
$$

$$
\begin{aligned}
{ }^{\mathrm{r}} \mathrm{B}_{3 p q} & =\left\langle\mathrm{d}_{33}^{-1} \mathrm{e}_{3 \mathrm{pq}}\right\rangle\left[\left\langle\mathrm{d}_{33}^{-1}\right\rangle-\left({ }^{\mathrm{r}} \mathrm{M} l_{3}\right)^{-1}\right]^{-1} \\
{ }^{\mathrm{r}} \mathrm{U}_{3 \mathrm{p}} & =\frac{{ }^{\mathrm{r}} \mathrm{Q} \cdot \mathrm{N}_{\mathrm{p}}-\left\langle\mathrm{P}^{-1} \mathrm{e}_{333}\right\rangle \mathrm{Z}_{\mathrm{p}}}{\left\langle\mathrm{P}^{-1} \mathrm{e}_{333}\right\rangle^{2}+{ }^{\mathrm{r}} \mathrm{F}^{\mathrm{r}} \mathrm{Q}}
\end{aligned}
$$

where $\quad \mathrm{P}=\mathrm{C}_{3333} \mathrm{~d}_{33}+\mathrm{e}_{333}^{2}, \quad{ }^{\mathrm{r}} \mathrm{F}=\left({ }^{\mathrm{r}} \mathrm{M} \cdot l_{3}\right)^{-1}+$ $\left\langle\mathrm{P}^{-1} \mathrm{C}_{3333}\right\rangle, \mathrm{N}_{\mathrm{p}}=\left\langle\mathrm{P}^{-1}\left(\mathrm{e}_{333} \mathrm{e}_{\mathrm{p} 33}+\mathrm{C}_{3333} \mathrm{~d}_{3 \mathrm{p}}\right)\right\rangle,{ }^{\mathrm{r}} \mathrm{Q}=$ $\left\langle\mathrm{P}^{-1} \mathrm{~d}_{33}\right\rangle+{ }^{\mathrm{r}} \mathrm{K}_{33}^{-1} l_{3}^{-1}, \quad \mathrm{Z}_{\mathrm{p}}=\left\langle\mathrm{P}^{-1}\left(\mathrm{e}_{\mathrm{p} 33} \mathrm{~d}_{33}-\mathrm{e}_{333} \mathrm{~d}_{3 \mathrm{p}}\right)\right\rangle$ and $\mathrm{H}_{\mathrm{pq}}=\left\langle\mathrm{P}^{-1}\left(\mathrm{~d}_{33} \mathrm{C}_{33 \mathrm{pq}}+\mathrm{e}_{333} \mathrm{e}_{3 \mathrm{pq}}\right)\right\rangle$.

Then, substituting Eqs. (27)-(30) into Eqs. (21) and (22) considering Eqs. (23) and (24), the expressions for the effective coefficients are found:

$$
\left\{\begin{array}{l}
\mathrm{C}_{\mathrm{ijpq}}^{*}=\left\langle\mathrm{C}_{\mathrm{ijpq}}\right\rangle+\sum_{\mathrm{r}=1}^{\mathrm{N}} \theta_{\mathrm{r}}\left\langle\mathrm{C}_{\mathrm{ijk} 3} \frac{\mathrm{d}^{\mathrm{r}} \Phi_{\mathrm{kpq}}}{\mathrm{dy}_{3}}+\mathrm{e}_{3 \mathrm{ij}} \frac{\mathrm{d}^{\mathrm{r}} \Psi_{\mathrm{pq}}}{\mathrm{dy}_{3}}\right\rangle, \\
\mathrm{e}_{\mathrm{pij}}^{*}=\left\langle\mathrm{e}_{\mathrm{pij}}\right\rangle+\sum_{\mathrm{r}=1}^{\mathrm{N}} \theta_{\mathrm{r}}\left\langle\mathrm{C}_{\mathrm{ijk} 3} \frac{\mathrm{d}^{\mathrm{r}} \Pi_{\mathrm{kp}}}{\mathrm{dy}_{3}}+\mathrm{e}_{3 \mathrm{ij}} \frac{\mathrm{d}^{\mathrm{r}} \Theta_{\mathrm{p}}}{\mathrm{dy}_{3}}\right\rangle, \\
\mathrm{d}_{\mathrm{ip}}^{*}=\left\langle\mathrm{d}_{\mathrm{ip}}\right\rangle-\sum_{\mathrm{r}=1}^{\mathrm{N}} \theta_{\mathrm{r}}\left\langle\mathrm{e}_{\mathrm{ik} 3} \frac{\mathrm{d}^{\mathrm{r}} \Pi_{\mathrm{kp}}}{\mathrm{dy}_{3}}-\mathrm{d}_{\mathrm{i} 3} \frac{\mathrm{d}^{\mathrm{r}} \Theta_{\mathrm{p}}}{\mathrm{dy}_{3}}\right\rangle .
\end{array}\right.
$$

To determine the effective coefficients [Eqs. (31)] the contribution of each $\mathrm{N}$ interface partition of the composite is needed. Then, finally, the functions $\mathrm{d}^{\mathrm{r}} \Phi_{\mathrm{kpq}} / \mathrm{dy}_{3}, \mathrm{~d}^{\mathrm{r}} \Psi_{\mathrm{pq}} / \mathrm{dy}_{3}, \mathrm{~d}^{r} \Pi_{\mathrm{kp}} / \mathrm{dy}_{3}$ and $\mathrm{d}^{\mathrm{r}} \Theta_{\mathrm{p}} / \mathrm{dy}_{3}$ can be written as: 


$$
\begin{aligned}
\mathrm{d}^{\mathrm{r}} \Psi_{\mathrm{pq}} / \mathrm{dy}_{3}= & \mathrm{P}^{-1}\left[\mathrm{e}_{333}\left({ }^{\mathrm{r}} \mathrm{A}_{33 \mathrm{pq}}-\mathrm{C}_{33 \mathrm{pq}}\right)\right. \\
& \left.-\mathrm{C}_{3333}\left({ }^{\mathrm{r}} \mathrm{B}_{3 \mathrm{pq}}-\mathrm{e}_{3 \mathrm{pq}}\right)\right], \\
\mathrm{d}^{\mathrm{r}} \Theta_{\mathrm{p}} / \mathrm{dy}_{3}= & \mathrm{P}^{-1}\left[\mathrm{e}_{333}\left({ }^{\mathrm{r}} \mathrm{J}_{\mathrm{p} 33}-\mathrm{e}_{\mathrm{p} 33}\right)+\mathrm{C}_{3333}\left({ }^{\mathrm{r}} \mathrm{U}_{3 \mathrm{p}}-\mathrm{d}_{3 \mathrm{p}}\right)\right],
\end{aligned}
$$

- If $\beta \neq 3$ :

$\mathrm{d}^{\mathrm{r}} \Phi_{\beta \mathrm{pq}} / \mathrm{dy}_{3}=\mathrm{C}_{\beta 3 \beta 3}^{-1}\left({ }^{\mathrm{r}} \mathrm{A}_{\beta 3 \mathrm{pq}}-\mathrm{C}_{\beta 3 \mathrm{pq}}\right)$,

$\mathrm{d}^{\mathrm{r}} \Pi_{\beta \mathrm{p}} / \mathrm{dy}_{3}=\mathrm{C}_{\beta 3 \beta 3}^{-1}\left({ }^{\mathrm{r}} \mathrm{J}_{\mathrm{p} \beta 3}-\mathrm{e}_{\mathrm{p} \beta 3}\right)$,

- If $\beta=3$ :

$$
\begin{aligned}
\mathrm{d}^{\mathrm{r}} \Phi_{3 \mathrm{pq}} / \mathrm{dy}_{3}= & \mathrm{P}^{-1}\left[\mathrm{~d}_{33}\left({ }^{\mathrm{r}} \mathrm{A}_{33 \mathrm{pq}}-\mathrm{C}_{33 \mathrm{pq}}\right)\right. \\
& \left.+e_{333}\left({ }^{\mathrm{r}} \mathrm{B}_{3 \mathrm{pq}}-\mathrm{e}_{3 \mathrm{pq}}\right)\right], \\
\mathrm{d}^{\mathrm{r}} \Pi_{3 \mathrm{p}} / \mathrm{dy}_{3}= & \mathrm{P}^{-1}\left[\mathrm{~d}_{33}\left({ }^{\mathrm{r}} \mathrm{J}_{\mathrm{p} 33}-\mathrm{e}_{\mathrm{p} 33}\right)-\mathrm{e}_{333}\left({ }^{\mathrm{r}} \mathrm{U}_{3 \mathrm{p}}-\mathrm{d}_{3 \mathrm{p}}\right)\right] .
\end{aligned}
$$

\section{Numerical results}

In the present work, it is investigated the influences of the non-uniform electrical and mechanical imperfect contacts on the effective piezoelectric moduli of layered composites considering different length fractions of the imperfection at the interface and volume fractions of the layer 1 (PZT-5A). Limit cases for the present model are verified, i.e., the analytical expressions [Eq. (31)] reproduce the material properties for each constituent when the volume fraction of layer 1 is equal to zero or one. Besides, the Eq. (31) can be reduced to the elastic case reported in Ref. [31], if we consider null piezoelectric properties and the partition number $\mathrm{N} \equiv 1$. On the other hand, as the mechanical and electrical imperfect parameters reach higher values (for example ${ }^{\mathrm{r}} \mathrm{K}_{\mathrm{ii}}=10^{9}$ and ${ }^{\mathrm{r}} \mathrm{M} \equiv 10^{6}$ ), the results of Ref. [30] for the perfect contact case are reproduced. These situations are also shown in Table 2 and Figs. 2, 3, 4.

A computational algorithm has been implemented for the illustration of the behavior of two layers composites (PZT-5A/Araldite) where the constituent parameters used in the calculations are given in
Table 1 and taken from Ref. [30]. Also, the permittivity of free space is $\mathrm{d}_{0}=8.85 \times 10^{-12} \mathrm{C}^{2} / \mathrm{Nm}^{2}$.

The numerical model is rapidly converging to local problem solution for any volume fraction of the composite constituents. For the case of the constituents reported in Table 1, the effect of the nonuniform imperfections (mechanical and electrical) can be more significantly detected for values of the PZT$5 \mathrm{~A}$ volume fraction higher than 0.75 according to the numerical results. Therefore, the results are only reported for PZT-5A volume fractions equal to 0.75 and higher. A bi-laminate composite PZT-5A/Araldite (ceramic/polymer) is a case where hard and soft constituents have a common interface. For the case, where the soft phase is dominant, most of the mechanical energy can relax in the soft phase and the quality of the contact does not play a dominant role. On the other side, when the hard phase is dominant, the properties of the interface have a more significant effect on the PZT-5A/Araldite composite properties.

A bi-laminated with the interface divided into two portions can be seen as a non-uniform imperfect contact conditions, where one part of the interface (Let us say the portion " 1 ") decreases its percentage from $\theta_{1}=100 \%$ to $\theta_{1}=0 \%$ with respect to the total area of the interface $\Gamma$. We can define the pair $\left[\theta_{1} ; \theta_{2}\right]$ where $\theta_{1}+\theta_{2}=100 \%$. Thus, the mechanical and electrical interface parameters for the first and second portion of the interface are taken as: ${ }^{1} \mathrm{~K}_{\mathrm{ii}}=10^{9},{ }^{1} \mathrm{M} \equiv 10^{6}$, and ${ }^{2} \mathrm{~K}_{\mathrm{ii}}={ }^{2} \mathrm{M}=50$.

In Table 2, it can be observed, the elastic $\left(\mathrm{C}_{1111}^{*}\right)$, piezoelectric $\left(\mathrm{e}_{333}^{*}\right)$ or dielectric $\left(\mathrm{d}_{33}^{*}\right)$ effective coefficients as a function of the layer volume fraction and the pair $\left[\theta_{1} ; \theta_{2}\right]$ that describes the non-uniformity of the imperfect contact. For the case where the first portion represents $100 \%$ of the interface, the numerical results reproduce the perfect contact reported in Ref. [30], whereas the first portion has $0 \%$ of the interface the numerical results reproduce the uniform imperfect contact reported in Ref. [25]. Also, when the portion one decreases its percentage, the calculated coefficients values move from perfect contact condition to uniform imperfect contact. Then, Table 2 describes a behavior that characterizes a transition between perfect contact and a uniform imperfect contact. The numerical coincidence of the two extreme cases $[100 ; 0]$ and $[0 ; 100]$ with the result 

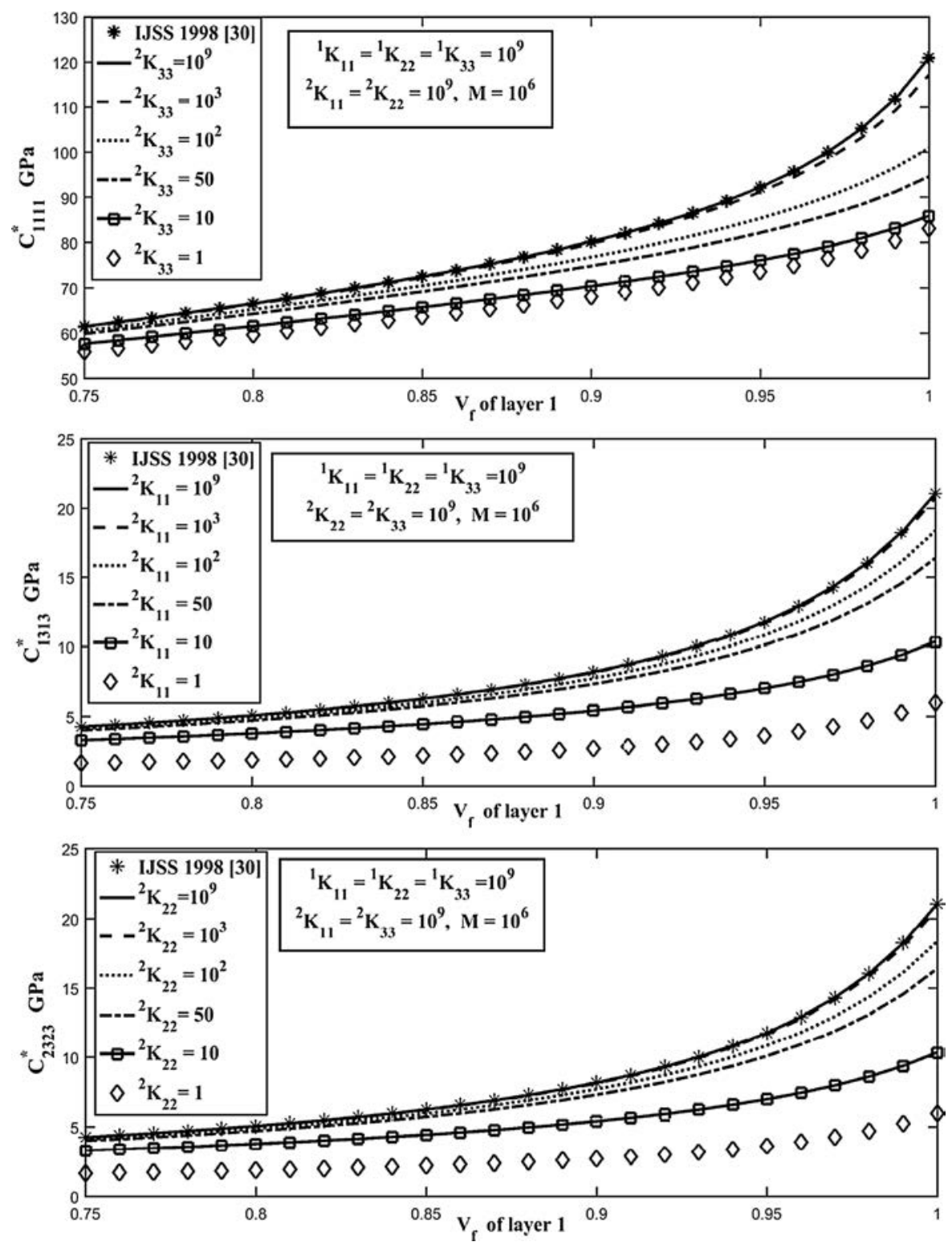

Fig. 2 Effect of the imperfection parameter on the effective elastic coefficients $C_{1111}^{*}, C_{1313}^{*}$ and $C_{2323}^{*}$ considering a bi-phase partition for the bi-laminate composite PZT-5A/Araldite

reported by Ref. [30] validates the analytical formulae obtained previously. It can be observed from Table 2 that as $\theta_{2}$ increases, the elastic, piezoelectric and dielectric coefficients decrease. This result can be qualified as physically congruent and expected because the second portion of the interface characterizes the imperfect contact.

A two layers composite with the interface divided into two portions is studied herein. In the first portion, we assume ${ }^{1} \mathrm{~K}_{\mathrm{ii}}=10^{9} \quad(\mathrm{ii}=11,22,33)$ and ${ }^{1} \mathrm{M}=10^{6}$. In the second part of the interface, the parameters are evaluated to be equal to ${ }^{2} \mathrm{~K}_{22}={ }^{2} \mathrm{~K}_{33}=$ $10^{9}$ and ${ }^{2} \mathrm{M}=10^{6}$ with the exception of ${ }^{2} \mathrm{~K}_{11}$ that runs from 1 to $10^{3}$, this way, we investigated the effect of ${ }^{2} \mathrm{~K}_{11}$ as can be seen in the ${ }^{2} \mathrm{~K}_{11}$ column of Table 3. In a similar way, we proceed with ${ }^{2} \mathrm{~K}_{22},{ }^{2} \mathrm{~K}_{33}$ and $\mathrm{M}$. The same results are obtained if the imperfection parameters of the portion 1 are investigated. 

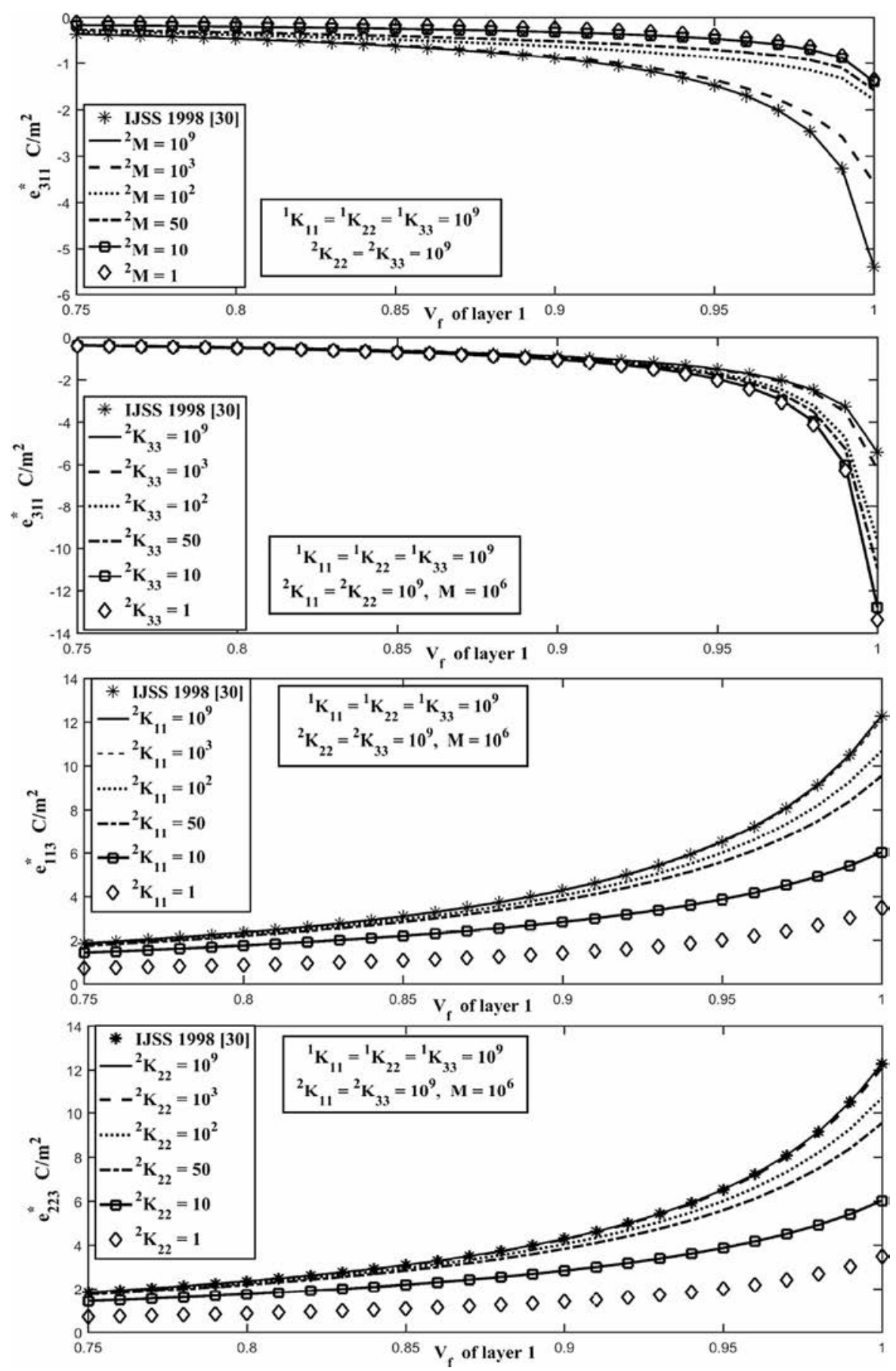

Fig. 3 Effect of the mechanical/electrical imperfection parameters on the effective piezoelectric coefficients $\mathrm{e}_{311}^{*}, \mathrm{e}_{113}^{*}$ and $\mathrm{e}_{223}^{*}$ for a bilaminate composite PZT-5A/Araldite

Finally, Table 3 shows the summary for the influence of the imperfection parameter on each effective coefficient, where three situations can be identified:
(1) the parameter significantly affects (Yes), (2) almost does not affect (Negligible) or (3) does not affect (Not) the value of the effective coefficients. 


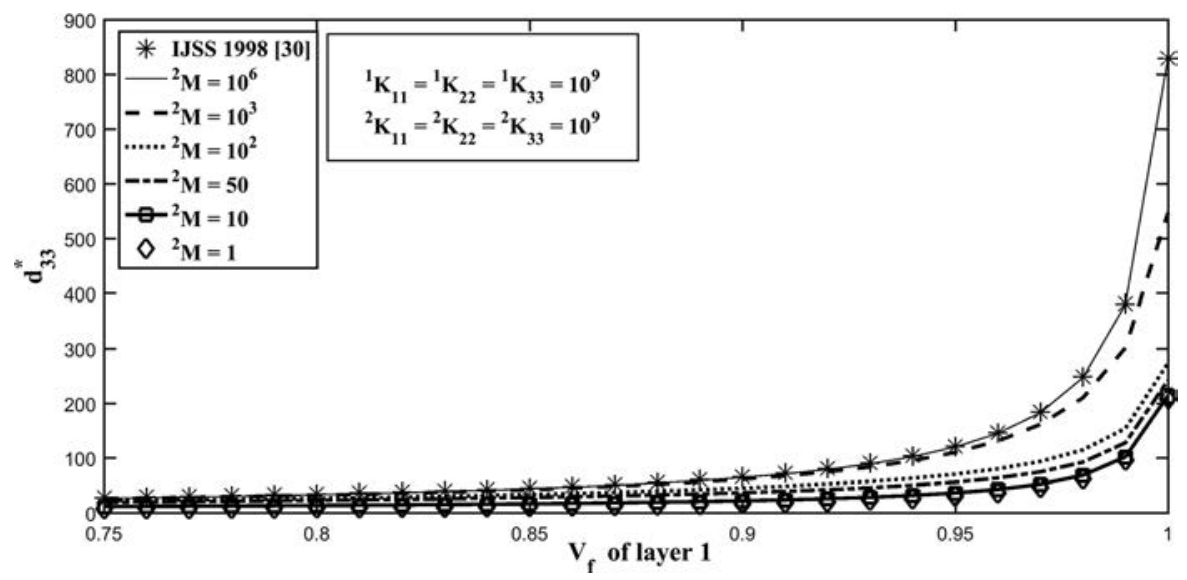

Fig. 4 Effect of the imperfection parameter on the effective dielectric coefficient $d_{33}^{*}$ considering a bi-phase partition for the bilaminated composite PZT-5A/Araldite

Table 1 Materials used in the computation

\begin{tabular}{lllllllllll}
\hline Dimension & $\mathrm{GPa}$ & \multicolumn{1}{c}{$\mathrm{C} / \mathrm{m}^{2}$} & \multicolumn{3}{c}{-} \\
\hline Parameters & $\mathrm{C}_{1111}$ & $\mathrm{C}_{1122}$ & $\mathrm{C}_{1133}$ & $\mathrm{C}_{3333}$ & $\mathrm{C}_{2323}$ & $\mathrm{e}_{311}$ & $\mathrm{e}_{333}$ & $\mathrm{e}_{223}$ & $\mathrm{~d}_{11} / \mathrm{d}_{0}$ & $\mathrm{~d}_{33} / \mathrm{d}_{0}$ \\
PZT-5A & 121 & 75.4 & 75.2 & 111 & 21.1 & -5.4 & 15.8 & 12.3 & 916 & 830 \\
Araldite & 5.46 & 2.94 & 2.94 & 5.46 & 1.26 & 0 & 0 & 0 & 7 & 7 \\
\hline
\end{tabular}

These results are congruent due to the mechanical imperfection parameters affect only the elastic and piezoelectric coefficients, while the dielectric coefficients are barely affected. A similar situation occurs with the electric imperfection parameter: it affects the piezoelectric and dielectric coefficients and barely affects the elastic coefficients.

In Figs. 2, 3 and 4 the effect caused by the variation of the imperfection parameter on the behavior of the effective elastic (Fig. 2), piezoelectric (Fig. 3) and dielectric (Fig. 4) coefficients is shown. Herein, a biphase partition for a bi-laminated PZT-5A/Araldita is considered. These results agree with Table 3. In Figs. 2 and 3, we illustrate the behavior of the effective coefficients $\mathrm{C}_{1111}^{*}$ and $\mathrm{e}_{311}^{*}$ because it is more significant than the coefficients $\mathrm{C}_{1122}^{*}, \mathrm{C}_{1133}^{*}, \mathrm{C}_{3333}^{*}$ and $\mathrm{e}_{333}^{*}$.

In Table 4, the effect of the interface partition on the effective coefficients $\mathrm{C}_{1133}^{*}, \mathrm{e}_{333}^{*}$ and $\mathrm{d}_{33}^{*}$ are also illustrated. Herein, a bi-laminated composite with a three partitioned interface $\left[\theta_{1} ; \theta_{2} ; \theta_{3}\right]$ is considered. Also, for each interphase portion, the imperfect parameters takes the values: ${ }^{1} \mathrm{~K}_{\mathrm{ii}}=100,{ }^{1} \mathrm{M}=100$, ${ }^{2} \mathrm{~K}_{\mathrm{ii}}=10^{9}, \quad{ }^{2} \mathrm{M}=10^{6}, \quad{ }^{3} \mathrm{~K}_{\mathrm{ii}}=50$, and ${ }^{3} \mathrm{M}=50$ $(\mathrm{ii}=11,22,33)$. From Table 4 , it is shown that
$\mathrm{C}_{1133}^{*}, \mathrm{e}_{333}^{*}$ and $\mathrm{d}_{33}^{*}$ values are always bounded between those values obtained when considering perfect and the uniform most imperfect contact, i.e., the case with the weakest contact $\left({ }^{3} \mathrm{~K}_{\mathrm{ii}}=50,{ }^{3} \mathrm{M}=50\right)$. It can also be seen, that the value of $\mathrm{C}_{1133}^{*}, \mathrm{e}_{333}^{*}$ and $\mathrm{d}_{33}^{*}$ are always affected by the type of partition that is proposed, for example: $[0 ; 100 ; 0]$ correspond to the perfect contact, $[0 ; 70 ; 30]$ and $[30 ; 70 ; 0]$ are expected to similarly behave due to for both cases, $30 \%$ of the contact area correspond to an imperfect contact. The slight difference between these cases is because, for the third partition, the imperfection parameters have lower values. The cases $[0 ; 0 ; 100]$ and $[100 ; 0 ; 0]$ represents two uniform imperfect contacts. As expected, the case $[50 ; 0 ; 50]$ can be visualized as an average between the two uniform imperfects. Again, the slight difference between them is a result of not having the same imperfection parameters.

Numerical results of Table 4 can reproduce the perfect and uniform imperfect contact reported in Refs. [25] and [30]; and in Table 2 as a limit case when one of the portions is null. It can be observed that the elastic, piezoelectric and dielectric coefficients are more sensitive to the imperfection parameters with the PZT-5A volume fraction between 0.9 and 1.0. As 
Table 2 Evolution of non-uniform imperfect contact for $\mathrm{C}_{1111}^{*}, \mathrm{e}_{333}^{*}$ and $\mathrm{d}_{33}^{*}$ considering a bi-phase partition for bi-laminate PZT-5A/ Araldite

\begin{tabular}{|c|c|c|c|c|c|c|c|}
\hline \multirow[t]{2}{*}{$\mathrm{V}_{\mathrm{f}}$} & \multirow[t]{2}{*}{ Perfect contact Ref. [30] } & \multicolumn{5}{|c|}{ Imperfect non-uniform contact } & \multirow{2}{*}{$\begin{array}{l}\text { Uniform imperfect } \\
\text { contact Ref. [25] }\end{array}$} \\
\hline & & {$[100 ; 0]$} & {$[75 ; 25]$} & {$[50 ; 50]$} & {$[25 ; 75]$} & {$[0 ; 100]$} & \\
\hline \multicolumn{8}{|c|}{ Elastic effective coefficient $\mathrm{C}_{1111}^{*}(\mathrm{GPa})$} \\
\hline 0.1 & 12.36484 & 12.36484 & 12.31532 & 12.26581 & 12.21630 & 12.16679 & 12.16679 \\
\hline 0.3 & 26.38717 & 26.38717 & 26.30220 & 26.21722 & 26.13224 & 26.04726 & 26.04726 \\
\hline 0.5 & 40.95013 & 40.95013 & 40.78501 & 40.61989 & 40.45477 & 40.28966 & 40.28966 \\
\hline 0.7 & 56.97634 & 56.97634 & 56.57170 & 56.16705 & 55.76241 & 55.35777 & 55.35777 \\
\hline 0.8 & 66.62882 & 66.62882 & 65.87556 & 65.12231 & 64.36905 & 63.61580 & 63.61580 \\
\hline 0.9 & 80.25864 & 80.25864 & 78.49033 & 76.72202 & 74.95372 & 73.18541 & 73.18541 \\
\hline 1 & 121.0000 & 120.9999 & 112.2711 & 103.5422 & 94.81337 & 86.08450 & 86.08450 \\
\hline \multicolumn{8}{|c|}{ Piezoelectric effective coefficient $\mathrm{e}_{333}^{*}\left(\mathrm{C} / \mathrm{m}^{2}\right)$} \\
\hline 0.1 & 0.000802 & 0.000802 & 0.000756 & 0.000711 & 0.000665 & 0.000619 & 0.000619 \\
\hline 0.3 & 0.003906 & 0.003906 & 0.003636 & 0.003365 & 0.003095 & 0.002825 & 0.002825 \\
\hline 0.5 & 0.012358 & 0.012358 & 0.011270 & 0.010182 & 0.009094 & 0.008006 & 0.008006 \\
\hline 0.7 & 0.044742 & 0.044741 & 0.039340 & 0.033939 & 0.028538 & 0.023137 & 0.023137 \\
\hline 0.8 & 0.105733 & 0.105730 & 0.074507 & 0.067231 & 0.058896 & 0.043285 & 0.043285 \\
\hline 0.9 & 0.379386 & 0.379361 & 0.307970 & 0.236579 & 0.165188 & 0.093797 & 0.093797 \\
\hline 1 & 15.80000 & 15.78690 & 11.90975 & 8.032601 & 4.155457 & 0.278307 & 0.278307 \\
\hline \multicolumn{8}{|c|}{ Dielectric effective coefficient $d_{33}^{*}$} \\
\hline 0.1 & 7.992715 & 7.770455 & 7.509174 & 7.247893 & 6.986611 & 6.725330 & 6.725330 \\
\hline 0.3 & 10.62890 & 9.963981 & 9.550080 & 9.136178 & 8.722276 & 8.308375 & 8.308375 \\
\hline 0.5 & 14.98597 & 13.88302 & 13.12879 & 12.37456 & 11.62032 & 10.86609 & 10.86609 \\
\hline 0.7 & 24.41096 & 22.88357 & 21.08742 & 19.29128 & 17.49513 & 15.69898 & 15.69898 \\
\hline 0.8 & 35.58161 & 33.85916 & 30.44151 & 27.02387 & 23.60622 & 20.18857 & 20.18857 \\
\hline 0.9 & 66.93087 & 65.06596 & 55.86808 & 46.67020 & 37.47232 & 28.27444 & 28.27444 \\
\hline 1 & 830.0000 & 829.3117 & 633.7748 & 438.2379 & 242.7010 & 47.16409 & 47.16409 \\
\hline
\end{tabular}

Table 3 Influence of imperfect parameters on the effective coefficients for non-uniform imperfect contacts

\begin{tabular}{lllll}
\hline Effective coefficients & ${ }^{2} \mathrm{~K}_{11}$ & ${ }^{2} \mathrm{~K}_{22}$ & ${ }^{2} \mathrm{~K}_{33}$ & ${ }^{2} \mathrm{M}$ \\
\hline $\mathrm{C}_{1111}^{*}, \mathrm{C}_{1122}^{*}, \mathrm{C}_{1133}^{*}, \mathrm{C}_{3333}^{*}$ & Not & Not & Yes & Negligible \\
$\mathrm{C}_{2323}^{*}$ & Not & Yes & Not & Not \\
$\mathrm{C}_{1313}^{*}$ & Yes & Not & Not & Not \\
$\mathrm{e}_{311}^{*}, \mathrm{e}_{333}^{*}$ & Not & Not & Yes & Yes \\
$\mathrm{e}_{223}^{*}$ & Not & Yes & Not & Not \\
$\mathrm{e}_{113}^{*}$ & Yes & Not & Not & Not \\
$\mathrm{d}_{11}^{*}$ & Negligible & Not & Not & Not \\
$\mathrm{d}_{22}^{*}$ & Not & Negligible & Not & Not \\
$\mathrm{d}_{33}^{*}$ & Not & Not & Negligible & Yes \\
\hline
\end{tabular}

mentioned above, this is a result of the increasing volume for the harder phase with higher dielectric constants.
With the present model, it is possible to study a composite with as many partitions for the interface as can be of interest. Herein, we only study the two- and 
Table 4 Effect of the partition at the interface on the effective coefficient considering a three-phase partition of the bi-laminate composite PZT-5A/Araldite

\begin{tabular}{|c|c|c|c|c|c|c|}
\hline$V_{f}$ & Perfect contact $[0 ; 100 ; 0]$ & {$[0 ; 70 ; 30]$} & {$[30 ; 70 ; 0]$} & $\begin{array}{l}\text { Uniform imperfect } \\
\text { contact }[0 ; 0 ; 100]\end{array}$ & {$[50 ; 0 ; 50]$} & $\begin{array}{l}\text { Uniform imperfect } \\
\text { contact }[100 ; 0 ; 0]\end{array}$ \\
\hline \multicolumn{7}{|c|}{ Elastic effective coefficient $\mathrm{C}_{1133}^{*}(\mathrm{GPa})$} \\
\hline 0.1 & 3.331176 & 3.274309 & 3.223565 & 2.972472 & 3.057045 & 3.141618 \\
\hline 0.3 & 4.425825 & 4.331593 & 4.249850 & 3.839242 & 3.975480 & 4.111718 \\
\hline 0.5 & 6.314348 & 6.135759 & 5.987945 & 5.226337 & 5.472694 & 5.719051 \\
\hline 0.7 & 10.35168 & 9.915773 & 9.587216 & 7.803455 & 8.351051 & 8.898646 \\
\hline 0.8 & 14.77787 & 13.95429 & 13.38914 & 10.14879 & 11.09070 & 12.03260 \\
\hline 0.9 & 25.04554 & 22.98375 & 21.80283 & 14.23651 & 16.20471 & 18.17292 \\
\hline 1 & 75.20008 & 63.29811 & 59.62219 & 23.27380 & 29.40032 & 35.52684 \\
\hline \multicolumn{7}{|c|}{ Piezoelectric effective coefficient $\mathrm{e}_{333}^{*}\left(\mathrm{C} / \mathrm{m}^{2}\right)$} \\
\hline 0.1 & 0.000802 & 0.000747 & 0.000772 & 0.000619 & 0.000661 & 0.000702 \\
\hline 0.3 & 0.003906 & 0.003581 & 0.003724 & 0.002825 & 0.003062 & 0.003300 \\
\hline 0.5 & 0.012358 & 0.011053 & 0.011599 & 0.008006 & 0.008917 & 0.009829 \\
\hline 0.7 & 0.044741 & 0.038260 & 0.040708 & 0.023137 & 0.027218 & 0.031299 \\
\hline 0.8 & 0.105730 & 0.086996 & 0.093304 & 0.043285 & 0.053798 & 0.064311 \\
\hline 0.9 & 0.379361 & 0.293692 & 0.315562 & 0.093797 & 0.130246 & 0.166696 \\
\hline 1 & 15.78690 & 11.13432 & 11.29207 & 0.278307 & 0.541231 & 0.804155 \\
\hline \multicolumn{7}{|c|}{ Dielectric effective coefficient $\mathrm{d}_{33}^{*}$} \\
\hline 0.1 & 7.770455 & 7.456918 & 7.602392 & 6.725330 & 6.967786 & 7.210243 \\
\hline 0.3 & 9.963981 & 9.467299 & 9.693151 & 8.308375 & 8.684794 & 9.061214 \\
\hline 0.5 & 13.88302 & 12.97794 & 13.37534 & 10.86609 & 11.52842 & 12.19075 \\
\hline 0.7 & 22.88357 & 20.72819 & 21.60526 & 15.69898 & 17.16075 & 18.62252 \\
\hline 0.8 & 33.85916 & 29.75798 & 31.29000 & 20.18857 & 22.74194 & 25.29531 \\
\hline 0.9 & 65.06596 & 54.02851 & 57.37220 & 28.27444 & 33.84727 & 39.42009 \\
\hline 1 & 829.3117 & 594.6674 & 607.2965 & 47.16409 & 68.21253 & 89.26097 \\
\hline
\end{tabular}

three portions cases with the sake of validating the model. The number of partitions at the interface may be use as way to characterize a more realistic interface between constituents.

\section{Conclusions}

In the present work, the formulae for effective properties of piezoelectric composites with $2 \mathrm{~mm}$ point group symmetry has been obtained, considering non-uniform imperfect adhesion between layers. This model is a generalization of those where perfect and uniform imperfect contact conditions are considered. These formulae have been numerically validated comparing with previous reported results and theoretical limit cases.

It can be observed that the effect of non-uniform imperfection on the interphase plays a very important role on the final properties. For a bi-laminate composite PZT-5A/Araldite, these effects are more intensively detected for PZT-5A volume fractions larger that 0.75 . The numerical values of the effective properties, where a non-uniform imperfect contact is considered, are always bounded between the values of the perfect and the uniform imperfect contact conditions. Anyway, this interval is large enough to make the portion combination effect capable of affecting the composite properties. For the PZT-5A/Araldite composite, the elastic constant runs from 15 to $75 \mathrm{GPa}$, the piezoelectric from 3 to $14 \mathrm{C} / \mathrm{m}^{2}$, and the dielectric one 
from 150 to 800 . Composite elastic properties are mainly affected by the mechanical imperfect conditions and dielectric properties only change because of the electrical imperfect contacts. Piezoelectric effective coefficients are always influenced by both mechanical and electrical imperfections.

The herein developed model can be used to estimate effective properties for a variety of situations concerning the quality of the interfaces between constituents. The possibility of simulating a number of partitions at the contact where each one has its imperfection parameter provides the tools to study more realistic contact regions.

Acknowledgements The authors JCLR and HCM thank the financial support for a sabbatical stay CONACYT 2018-1 performed at the Autonomous University of Ciudad Juarez. The author YEA gratefully acknowledges the Program of Postdoctoral Scholarships of DGAPA from UNAM (20192020), México. HCM and YEA are grateful to the support of the CONACYT Basic science Grant A1-S-9232. The author RRR thanks to Mathematic and Mechanic Department, IIMAS and PREI-DGAPA at UNAM (2018), for the support to his research project. FJS thanks the funding of DGAPA, UNAM. This work was supported by the project PAPIIT-DGAPA-UNAM IA100919. The authors HBS and VT are thankful to Coordination for the Improvement of the Higher Level Personnel (CAPES/PNPD), National Council for Scientific and Technological Development (CNPq Process Number: 401170/2014-4 and 310094/2015-1). The authors are thankful to Ramiro Chávez Tovar and Ana Pérez Arteaga for computational assistance.

\section{Compliance with ethical standards}

Conflict of interest The authors declare that they have no conflict of interest.

\section{References}

1. Duan HL, Yi X, Huang ZP, Wang J (2007) A unified scheme for prediction of effective moduli of multiphase composites with interface effects. Part I: theoretical framework. Mech Mater 39(1):81-93. https://doi.org/10. 1016/j.mechmat.2006.02.009

2. Duan HL, Yi X, Huang ZP, Wang J (2007) A unified scheme for prediction of effective moduli of multiphase composites with interface effects. Part II: application and scaling laws. Mech Mater 39(1):94-103. https://doi.org/10. 1016/j.mechmat.2006.02.010

3. Rodríguez-Ramos R, Guinovart-Díaz R, López-Realpozo JC, Bravo-Castillero J, Sabina FJ (2010) Influence of imperfect elastic contact condition on the antiplane effective properties of piezoelectric fibrous composites. Arch
Appl Mech 80(4):377-388. https://doi.org/10.1007/s00419009-0320-3

4. López-Realpozo JC, Rodríguez-Ramos R, Guinovart-Díaz R, Bravo-Castillero J, Sabina FJ (2011) Transport properties in fibrous elastic rhombic composite with imperfect contact condition. Int J Mech Sci 53(2):98-107. https://doi.org/10. 1016/j.ijmecsci.2010.11.006

5. Rodríguez-Ramos R, Medeiros R, Guinovart-Díaz R, López-Realpozo JC, Bravo-Castillero J, Otero JA, Tita V (2013) Different approaches for calculating the effective elastic properties in composite materials under imperfect contact adherence. Compos Struct 99:264-275. https://doi. org/10.1016/j.compstruct.2012.11.040

6. Wah T (1973) Stress distribution in a bonded anisotropic lap joint. J Eng Mater Technol ASME 95:174-181. https://doi. org/10.1115/1.3443146

7. Ojalvo IU, Eidinoff HL (1978) Bond thickness effects upon stresses in single-lap adhesive joints. AIAA J 16:204-211. https://doi.org/10.2514/3.60878

8. Delale F, Erdogan F, Aydinoglu MN (1981) Stresses in adhesively bonded joints: a closed-form solution. J Compos Mater 15:249-271. https://doi.org/10.1177/ 002199838101500305

9. Chen D, Cheng S (1983) An analysis of adhesive-bonded single-lap joints. J Appl Mech ASME 50:109-115. https:// doi.org/10.1115/1.3166976

10. Adams RD, Comyn J, Wake WC (1984) Structural adhesive joints in engineering. Elsevier Applied Science, London and New York

11. Higgins A (2000) Adhesive bonding of aircraft structures. Int J Adhes 20:367-376. https://doi.org/10.1016/S01437496(00)00006-3

12. Knollman GC (1985) Variation of shear modulus through the interfacial bond zone of an adhesive. Int $\mathrm{J}$ Adhes 5:137-141. https://doi.org/10.1016/0143-7496(85)90055-7

13. Safavi-Ardebili V, Sinclair AN, Spelt JK (1997) Experimental investigation of the interphase in an epoxy-aluminum system. J Adhes 62:93-111. https://doi.org/10.1080/ 00218469708014564

14. Krüeger JK, Possart W, Bactavachalou R, Müeller U, Britz T, Sanctuary R, Alnot P (2004) Gradient of the mechanical modulus in glass-epoxy-metal joints as measured by Brillouin microscopy. J Adhes 80:585-599. https://doi.org/10. 1080/00218460490476973

15. Chung J, Munz M, Sturm H (2007) Stiffness variation in the interphase of amine-cured epoxy adjacent to copper microstructures. Surf Interface Anal 39:624-633. https:// doi.org/10.1002/sia.2571

16. Parlevliet PP, Bersee HEN, Beukers A (2007) Residual stresses in thermoplastic composites. A study of the literature. Part II: experimental techniques. Compos Part A Appl Sci Manuf 38:651-665. https://doi.org/10.1016/j. compositesa.2006.07.002

17. Walpole LJ (1978) A coated inclusion in an elastic medium. Math Proc Camb Philos Soc 83:495-506. https://doi.org/10. 1017/S0305004100054773

18. Maurini C, Pouget J, dell'Isola F (2006) Extension of the Euler-Bernoulli model of piezoelectric laminates to include $3 \mathrm{D}$ effects via a mixed approach. Comput Struct 84(22-23):1438-1458. https://doi.org/10.1016/j. compstruc.2006.01.016 
19. Bensoussan A, Lions JL, Papanicolaou G (1978) Asymptotic analysis for periodic structures. North-Holland, Amsterdam

20. Sanchez-Palencia E (1980) Non homogenous media and vibration theory. Lecture notes in physics. Springer, Berlin

21. Galka A, Telega JJ, Wojnar R (1996) Some computational aspects of homogenization of thermopiezoelectric composites. Comput Assist Mech Eng Sci 3:133-154

22. Oleinik OA, Panasenko GP (1983) Homogenization and asymptotic expansions for solutions of the elasticity system with rapidly oscillating periodic coefficients. Appl Anal 15:15-32. https://doi.org/10.1080/00036818308839437

23. Bakhvalov NS, Panasenko GP (1989) Homogenization averaging processes in periodic media. Kluwer Academic Publishers, Dordrecht

24. Cherednichenko KD, Evans JA (2016) Full two-scale asymptotic expansion and higher-order constitutive laws in the homogenization of the system of quasi-static Maxwell equation. SIAM Multiscale Model Simul 14:1513-1539. https://doi.org/10.1137/15M1042103

25. Brito-Santana H, Medeiros R, Rodríguez-Ramos R, Tita V (2016) Different interface models for calculating the effective properties in piezoelectric composite materials with imperfect fiber-matrix adhesion. Compos Struct 151:70-80. https://doi.org/10.1016/j.compstruct.2016.02. 003

26. Andrianov IV, Bolshakov VI, Danishevs'kyy VV, Weichert D (2008) Higher order asymptotic homogenization and wave propagation in periodic composite materials. Proc $\mathrm{R}$
Soc A 464:1181-1201. https://doi.org/10.1098/rspa.2007. 0267

27. Lebon F, Dumont S, Rizzoni R, López-Realpozo JC, Guinovart-Díaz R, Rodríguez-Ramos R, Bravo-Castillero J, Sabina FJ (2016) Soft and hard anisotropic interface in composite materials. Compos Part B Eng 90:58-68. https:// doi.org/10.1016/j.compositesb.2015.12.003

28. Wang X, Schiavone P (2017) A circular inhomogeneity with a mixed-type imperfect interface in anti-plane shear. Appl Math Model 43:538-547. https://doi.org/10.1016/j.apm. 2016.11.035

29. Sabiston T, Mohammadi M, Cherkaoui M, Levesque J, Inal K (2016) Micromechanics for a long fibre reinforced composite model with a functionally graded interphase. Compos Part B Eng 84:188-199. https://doi.org/10.1016/j. compositesb.2015.08.070

30. Bravo-Castillero J, Otero-Hernandez JA, Rodríguez-Ramos R (1998) Asymptotic homogenization methods of laminated piezocomposite materials. Int J Solid Struct 5-6:527-541. https://doi.org/10.1016/S0020-7683(97)00028-0

31. Brito-Santana H, Medeiros R, Mendes-Ferreira AJ, Rodríguez-Ramos R, Tita V (2018) Effective elastic properties of layered composites considering non-uniform imperfect adhesion. Appl Math Model 59:183-204. https://doi.org/10. 1016/j.apm.2018.01.009

Publisher's Note Springer Nature remains neutral with regard to jurisdictional claims in published maps and institutional affiliations. 